\title{
ORIGINAL ARTICLE Multi-polygenic score approach to trait prediction
}

\author{
E Krapohl ${ }^{1}$, H Patel ${ }^{2,3}$, S Newhouse ${ }^{2,3,4}$, CJ Curtis ${ }^{1,2}$, S von Stumm ${ }^{5}$, PS Dale ${ }^{6}$, D Zabaneh ${ }^{1}$, G Breen ${ }^{1,2}$, PF O’Reilly ${ }^{1}$ and R Plomin ${ }^{1}$
}

A primary goal of polygenic scores, which aggregate the effects of thousands of trait-associated DNA variants discovered in genome-wide association studies (GWASs), is to estimate individual-specific genetic propensities and predict outcomes. This is typically achieved using a single polygenic score, but here we use a multi-polygenic score (MPS) approach to increase predictive power by exploiting the joint power of multiple discovery GWASs, without assumptions about the relationships among predictors. We used summary statistics of 81 well-powered GWASs of cognitive, medical and anthropometric traits to predict three core developmental outcomes in our independent target sample: educational achievement, body mass index (BMI) and general cognitive ability. We used regularized regression with repeated cross-validation to select from and estimate contributions of 81 polygenic scores in a UK representative sample of 6710 unrelated adolescents. The MPS approach predicted $10.9 \%$ variance in educational achievement, $4.8 \%$ in general cognitive ability and 5.4\% in BMI in an independent test set, predicting $1.1 \%, 1.1 \%$, and $1.6 \%$ more variance than the best single-score predictions. As other relevant GWA analyses are reported, they can be incorporated in MPS models to maximize phenotype prediction. The MPS approach should be useful in research with modest sample sizes to investigate developmental, multivariate and gene-environment interplay issues and, eventually, in clinical settings to predict and prevent problems using personalized interventions.

Molecular Psychiatry (2018) 23, 1368-1374; doi:10.1038/mp.2017.163; published online 8 August 2017

\section{INTRODUCTION}

Genome-wide association studies (GWASs) have been successful in identifying thousands of associations for hundreds of complex traits and common disorders. ${ }^{1}$ One use of GWAS results is to understand biological pathways between genotypes and phenotypes. Another use, the focus of the present research, is to estimate genetic propensities of individuals to predict individuals' future problems and potential and, eventually, to develop personalized interventions that meet individual medical, psychiatric and educational needs. Both goals have been hindered by the ubiquitous GWA finding that the largest effect sizes are extremely small. $^{2}$ For example, the largest population effect sizes found for common variants in height or body mass index (BMI) account for only $\sim 1 \%$ of the variance. ${ }^{3,4}$ We know empirically that the vast majority of common genetic variants for most traits have a markedly lower effect than $1 \%{ }^{2}$

The highly polygenic nature of complex traits and common disorders poses an immense challenge for understanding the biological mechanisms linking single variants with phenotypes. However, when the priority is phenotypic prediction, polygenic scores can be used to aggregate the effects of many DNA variants in order to investigate their joint predictive power. ${ }^{5,6}$ Rather than just using single-nucleotide polymorphisms (SNPs) that reach genome-wide significance, a recent development is to aggregate a much larger number of SNPs, weighted by their GWA effect size estimate, as long as together they increase the prediction in an independent sample, even if some SNPs have no real effect. ${ }^{7}$ For example, for height, a polygenic score that aggregates the effects of 2000 SNPs accounts for $21 \%$ of the variance of height in independent samples. ${ }^{3}$

The other defining characteristic of complex traits and common disorders is the abundance of genetic correlations between them. There is consistent evidence for genetic correlations between psychiatric disorders, between anthropometric traits and between educational and cognitive traits, as well as for genetic correlations across these categories. ${ }^{8-11}$

Genetic correlation can arise from pleiotropy, the phenomenon of multiple traits being associated with the same gene or genetic variant. $^{8}$ Genetic correlation can also reflect shared biological pathways or more indirect linkage. ${ }^{12}$ Regardless of its cause, genetic correlation between different traits means that a polygenic score based on one trait can predict a different outcome trait, with predictive accuracy a function of the shared genetic signal between them. Therefore, when the aim is prediction, genetic correlation can be exploited for trait prediction while remaining agnostic to the underlying mechanisms.

A primary goal of polygenic scores, which aggregate the effects of thousands of trait-associated genetic variants discovered in GWAS, is to estimate individual-specific genetic propensities. This is typically achieved using a single polygenic score, but here we use an approach to increase predictive power by exploiting the joint power of multiple discovery GWASs. We use a multipolygenic score (MPS) approach that exploits genetic correlations between the outcome trait and a multitude of traits by using the joint predictive power of multiple polygenic scores in one regression model.

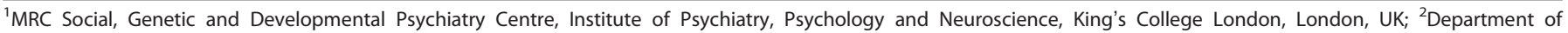

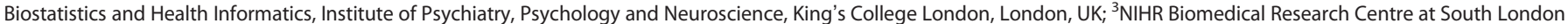

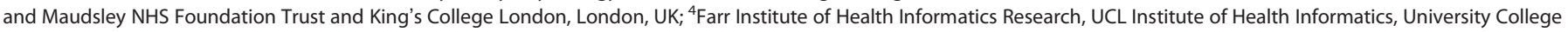

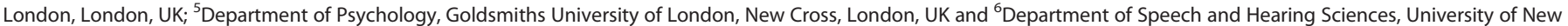

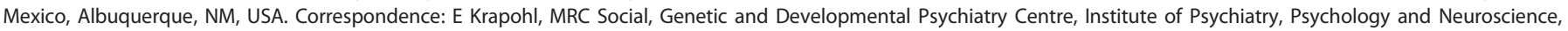
King's College London, 16 DeCrespigny Park, London SE5 8AF, UK.

E-mail: eva.krapohl@kcl.ac.uk

Received 19 October 2016; revised 12 May 2017; accepted 20 June 2017; published online 8 August 2017 
We selected GWASs from a centralized repository of summary statistics - based on their statistical power and regardless of prior evidence for association with the outcomes-to predict three core developmental outcomes in our independent target sample: educational achievement, BMI, and general cognitive ability. Using repeated cross-validation, we trained and validated the prediction models using elastic net regularized regression, a multiple regression model suited to deal with a large number of correlated predictors while preventing overfitting. ${ }^{13}$ We subsequently tested how well these models predict outcomes in an independent test set.

Here, we employ a MPS approach that uses publicly available GWAS summary statistics to estimate individual-level genetic propensities and predict developmental outcomes in an independent target sample. This stands in contrast to multi-trait approaches that rely on access to individual-level data in the discovery data sets because they make use of a method from animal breeding in which the total genetic effect ('breeding value') of each individual in a discovery data set is estimated from the best linear unbiased predictor in a multi-trait random-effects model that can be used for individual-level prediction in the validation data sets. These multi-trait methods are not applicable to GWAS summary statistics when genotype data are unavailable because of privacy or logistical constraints that are frequently the case.

The declared aim of the current MPS approach is to maximize prediction of developmental outcomes, rather than investigating their etiology. This stands in contrast to multi-trait meta-analytic approaches of GWAS summary statistics that relies on substantial and consistent correlations between discovery GWASs and whose main aim is variant discovery. ${ }^{14-17}$ The current MPS approach allows for, but does not require, correlation among polygenic predictors.

\section{MATERIALS AND METHODS}

\section{Sample}

The target sample comprised genome-wide SNP and phenotypic data from 6710 unrelated adolescents drawn from the UK representative Twins Early Development Study (TEDS). TEDS is a multivariate longitudinal study that recruited over 11000 twin pairs born in England and Wales in 1994, 1995 and 1996. Both the overall TEDS sample and the genotyped subsample have been shown to be representative of the UK population. ${ }^{18-20}$ The project received approval from the Institute of Psychiatry ethics committee (05/Q0706/228) and parental consent was obtained before data collection. We processed the genotypes for the 6710 individuals using stringent quality control procedures followed by imputation of SNPs using the Haplotype Reference Consortium reference panel ${ }^{21}$ (Supplementary Methods S1).

\section{Predictors}

Discovery data sets: GWAS summary statistics. We selected GWAS summary statistics from $L D$ hub, a centralized repository for summary statistics ${ }^{22}$ based on their statistical power-regardless of prior evidence for association with our outcome traits. Specifically, we included 81 GWAS summary statistics that were either publically downloadable or obtained via correspondence and had a linkage disequilibrium (LD) score ${ }^{23}$ heritability $z$-score $>5$, indexing good statistical power (which is a function of variance explained and sample size). Supplementary Table S1 provides details of all GWAS summary statistics included in our analyses.

The published version of the child IQ GWAS included the present target sample of TEDS. Therefore, to avoid bias, the present analyses used summary statistics from a rerun of the GWAS meta-analysis excluding TEDS.

Polygenic scores. We created 81 genome-wide polygenic scores for each of the 6710 individuals in the TEDS sample using summary statistics from the GWAS described above (Supplementary Table S1). After quality control (Supplementary Methods S1), the study data included 7581516 genotyped or well-imputed (info $>0.70$ ) SNPs. These were quality controlled and coordinated with each of the summary statistics, respectively, by excluding markers due to nucleotide inconsistencies or low minor allele frequency $(<1 \%)$. Number of markers before and after quality control and coordination with the study data are listed in Supplementary Table S1.

We constructed polygenic scores as the weighted sums of each individual's trait-associated alleles across all SNPs. We used LDpred ${ }^{24}$ to construct the scores. LDpred uses a prior on the markers' effect sizes and adjusts summary statistics for LD between markers. Scores were standardized and adjusted for 30 principal components. More details on the construction of the polygenic scores are provided in Supplementary Methods S2.

\section{Outcomes}

To illustrate the MPS approach, we selected three key developmental outcomes:

Educational achievement operationalized as the mean grade of the three compulsory subjects (Mathematics, English and Science) attained on the standardized United Kingdom General Certificate of Secondary Education (GCSE), taken by almost all (>99\%) pupils at the end of compulsory education at age 16 years.

General cognitive ability at age 12 years assessed by two verbal and two nonverbal cognitive standardized tests.

BMI at age 9 years that was age and sex adjusted using external reference data.

Supplementary Methods S3 and Figure S1 contain detailed descriptions of the three measures.

\section{Models}

Single-polygenic score models. To estimate the separate prediction of each predictor, we fit a series of simple linear regression models for each of the 81 polygenic scores and each of the 3 outcomes. For each GWAS-outcome combination, three models were run using polygenic scores created with Gaussian mixture weights of $1,0.1$ and 0.01 , respectively. The model that explained the most variance in the outcome (that is, largest cross-validated $R^{2}$ in training data) was then entered into the multi-score model. These simple linear regression models were fit and validated in repeated 10-fold cross-validation (see section below for details) using the $\mathrm{Im}$ function implemented within the caret R package..$^{25}$ Based on consistent evidence for extensive genetic correlations across complex traits and disorders, rather than summing up, the predictions of the single-score models were expected to substantially overlap.

MPS models. We used the MPS model to estimate the joint prediction of the 81 polygenic scores as well as the ranking of predictors by the magnitude of their contribution to predicting the outcome.

Conventional multiple linear regression models in the presence of a large number of predictors are subject to overfitting, and stepwise regression suffers from upward-biased coefficients and $R^{2}$ (see, for example, Tibshirani ${ }^{26}$ ). We used elastic net regularized regression ${ }^{13}$ to predict outcomes and by selecting predictors and estimating their contribution to the prediction. Regularized regression models are general linear models that employ strict penalties to prevent overfitting. Elastic net allows for estimating the joint predictive ability of a large number of variables while preventing overfitting. Elastic net uses a linear combination of two regularization techniques, L2 regularization (used in ridge regression) and L1 regularization (used in LASSO (least absolute shrinkage and selection operator)) by simultaneously implementing variable selection (that is, dropping/retaining variables) and continuous shrinkage (that is, penalizing coefficients for overfitting); and it efficiently deals with multicollinearity by selecting or dropping groups of correlated variables. ${ }^{13,27}$

Elastic net overcomes the limitation of LASSO that tends to select one variable from a group of correlated predictors and to ignore the others. In situations where predictors are non-independent or correlated (for example, sharing genetic signal or discovery cohorts) the elastic net has the advantage of including automatically all the highly correlated variables in the group (grouping effect). ${ }^{13,27,28}$

Final model coefficients are analogous to a conventional multiple linear regression output that allows for a ranking of predictors by the magnitude of their contribution to predicting the outcome. Overall variance explained by the model is indexed by the coefficient of determination, $R^{2}$.

We used glmnet $\mathrm{R}$ package $\mathrm{e}^{15-17}$ implemented within caret $\mathrm{R}$ package ${ }^{25}$ to conduct a series of linear elastic net regularized regressions and select polygenic predictors leading to an optimized final model for each 
outcome. Elastic net regularized regression employs two hyperparameters, alpha and lambda. ${ }^{13}$ As recommended to achieve optimized balance between variance explained and minimum bias, we fit models to tune over both alpha and lambda parameter values in repeated 10 -fold crossvalidation. $^{29}$.

\section{Model training and testing}

Generally, a predictive model is considered powerful when the model is capable of predicting outcomes in 'unseen' data with high accuracy. The performance of a model can therefore be evaluated by testing how well it predicts phenotypes of individuals whose data were not included in the construction of the prediction model.

Each model described in the preceding section was trained and tested using the following three-step strategy:

Data splitting. We randomly split the data set into a separate training set and test set $(60 \%$ train, $40 \%$ test).

Model training. We used repeated cross-validation on the training set to train and optimize the model via validation.

Model testing and comparison. We applied the final model to the independent test set to obtain an unbiased estimate of model performance.

Model training. The training set was used to train and validate the model, this included hyperparameter tuning for the elastic net models. In order to optimize the balance between variance explained and minimum bias, we tested each model in 10-fold cross-validation with resampling. ${ }^{29}$ We split the training data randomly into 10 equal-sized subsets, using 9 subsets to train the model and the remaining subset as validation. The crossvalidation process was repeated 10 times, with each of the 10 subsamples used once as the validation data.

Although cross-validation has been shown to produce nearly unbiased estimates of accuracy, variability of these estimates can be reduced by bootstrap methods, wherein available data are repeatedly sampled with replacement in order to mimic the drawing of future random sampling. ${ }^{30,31}$ Therefore, to minimize variation across validation data sets, we repeated the 10-fold cross-validation 100 times with random data set partitions. $^{32}$

The optimized or 'final' model is chosen based on the largest performance value (or smallest mean squared error). Predictors retained within the model and standardized coefficients index whether, and to what extent, they contribute to predicting the outcome. Model performance for the repeated cross-validation in the training set was summarized as mean-cv- $R_{\text {train }}^{2}$ from the resampling distribution.

Model testing and comparison. To obtain unbiased estimates of model performance, we used the parameters from the final model obtained from the repeated cross-validation in the training set to predict outcomes (that is, educational achievement, BMI and general cognitive ability) in the independent test set. To index prediction accuracy, we used the coefficient of determination, in the following referred to as $R_{\text {test. }}^{2}$. Differences between mean-cv- $R_{\text {train }}^{2}$ and $R_{\text {test }}^{2}$ provide an index of out-ofsample error.

We used permutation to test the statistical significance of the difference in predictions between the MPS and the best single-score model. To test the null hypothesis of exchangeability of models, $H_{0}:{ }_{\text {MPS }} R_{\text {test }}^{2}=$ best-single${ }_{\text {score }} R_{\text {test }}^{2}$, we compared the observed ${ }_{\text {diff }} R_{\text {test }}^{2}$ (MPs $R_{\text {test }}^{2}-$ best-single-score $R_{\text {test }}^{2}$ ) against an empirical null distribution of no difference in predictions between the MPS and the best single-score model. We tested the exchangeability of models by randomly selecting either the MPS or the best single-score model to generate predictions. We then calculated the difference in $R^{2}$ for two models with shuffled predictions. The process was repeated 100000 times, generating an empirical null distribution of diff $R^{2}$ under exchangeability of model predictions.

If the null hypothesis of no difference between models is true, it would not matter if we randomly exchange the model used for generating predictions. However, if the observed ${ }_{\text {diff }} R_{\text {test }}^{2}$ value falls outside of those obtained when randomly exchanging models, this represents evidence against the null hypothesis of no difference in prediction between models. The statistical significance, as expressed in an empirical $P$-value, is calculated as the fraction of permutation values that are at least as extreme as the original diff $R_{\text {test }}^{2}$ statistic observed in nonpermuted data.

\section{RESULTS}

MPS predictions

The MPS models showed better prediction in the independent test set than the best single-score models. The best single-score models were the large 2016 GWAS of years of education predicting $9.8 \%$ of the variance in educational achievement and $3.6 \%$ in general cognitive ability in the test set. For BMI, Obesity class 1 achieved the best single-score prediction, explaining 3.8\% of the variance. (See Supplementary Table S2 for full single-score models results; see Supplementary Figure S2 for a visual overview of the single-score model results.) The MPS models explained $10.9 \%$ variance in educational achievement, $4.8 \%$ in cognitive ability and $5.4 \%$ in BMI in the test set. The improvement in variance explained compared with the best single-score models was $1.1 \%(P=4 \mathrm{e}-03), 1.1 \%(P=2 \mathrm{e}-03)$ and $1.6 \%(P=1 \mathrm{e}-04)$, respectively.

Figures 1a-c show the polygenic predictors selected during training of the MPS models and their standardized coefficients. The ranking of predictors provides an index for their contributions to prediction. Analogous to conventional multiple regression, a standardized coefficient represents the contribution of the predictor to the outcome when adjusting for all other variables in the model.

The model predicting educational achievement retained 12 polygenic predictors (Figure 1a). Cognitive and socioeconomic polygenic scores took the top ranks. However, the psychiatric cross-disorder polygenic score, which aggregates genetic risk for bipolar disorder, schizophrenia, major depressive disorder, autism and attention deficit hyperactivity disorder, and the score for depressive symptoms in the general population were also retained by the model. The scores for Homeostasis Model Assessment of $\beta$-cell function, an index of $\beta$-cell function, and for coronary artery disease also contributed to prediction of educational achievement.

The MPS model predicting cognitive ability selected 10 polygenic scores during cross-validation (Figure 1b). The strongest contributions to prediction came from cognitive and socioeconomic variables. Contributions from the psychiatric realm came from major depressive disorder, autism spectrum disorder and bipolar disorder, with the latter two having positive association with cognitive ability.

The MPS model predicting BMI retained 28 polygenic scores (Figure 1c). The top three strongest predictions came from obesity-related variables. Ranks four and five were taken by coronary artery disease and age at menarche (negative association). The sixth strongest predictor for children's BMI was the polygenic score based on the GWAS of mean caudate nucleus volume that plays a role in various non-motor functions including procedural and associative learning and inhibitory action control. $^{33-36}$ Other predictors included ulcerative colitis, leptin and neuroticism.

\section{Stratification by MPS}

We examined the phenotypic values by quantile of the MPS distribution. Figures $2 \mathrm{a}-\mathrm{c}$ plot the observed outcomes against the predictions by the MPS model in the test set. In general, the quantile results were roughly linear.

Figure 2a shows quantile results for mean exam grades. Individuals in the top $10 \%$ of the MPS distribution on average achieved an ' $A$ ' mean grade (across the three subjects Mathematics, English and Science), whereas individuals in the bottom $10 \%$ MPS distribution achieved a ' $C$ ' mean grade on average (top $10 \%$ mean $=9.74$; bottom $10 \%$ mean $=8.33\left(11=A^{*}, 10=A, 9=B\right.$, $8=C, 7=D, 6=E, 5=F, 4=G, 0=$ failed). Cohen's $d$ was $1.20(95 \%$ confidence interval $0.99-1.41$ ) suggesting that $88 \%$ of the top $10 \%$ MPS group had a mean grade above that of the bottom $10 \%$ group, and there is an $80 \%$ probability that a person picked at 
a

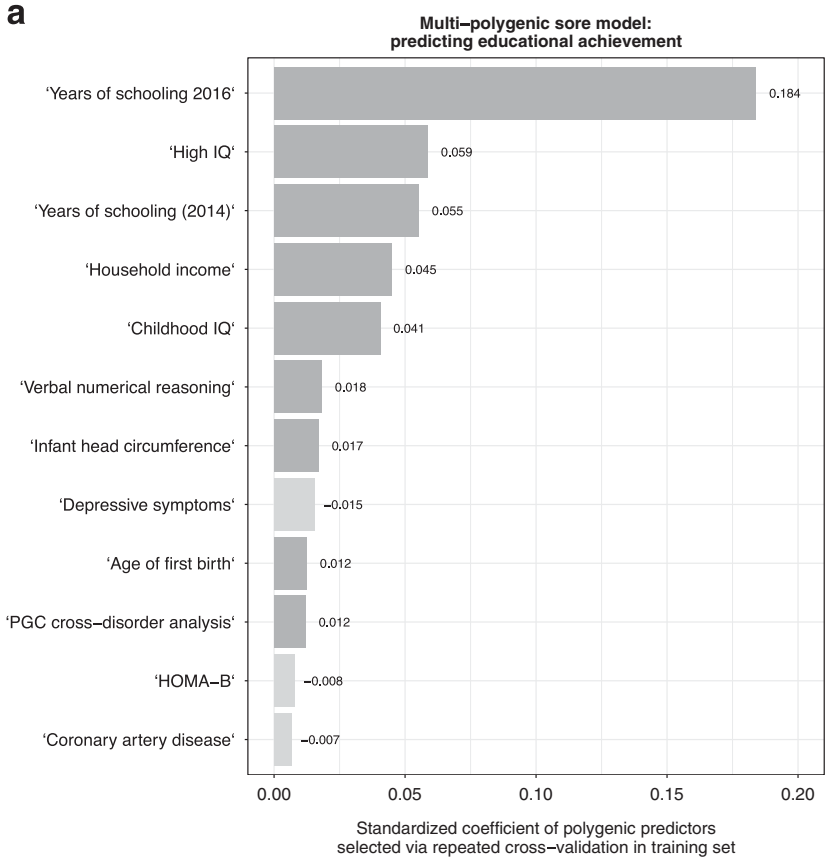

b

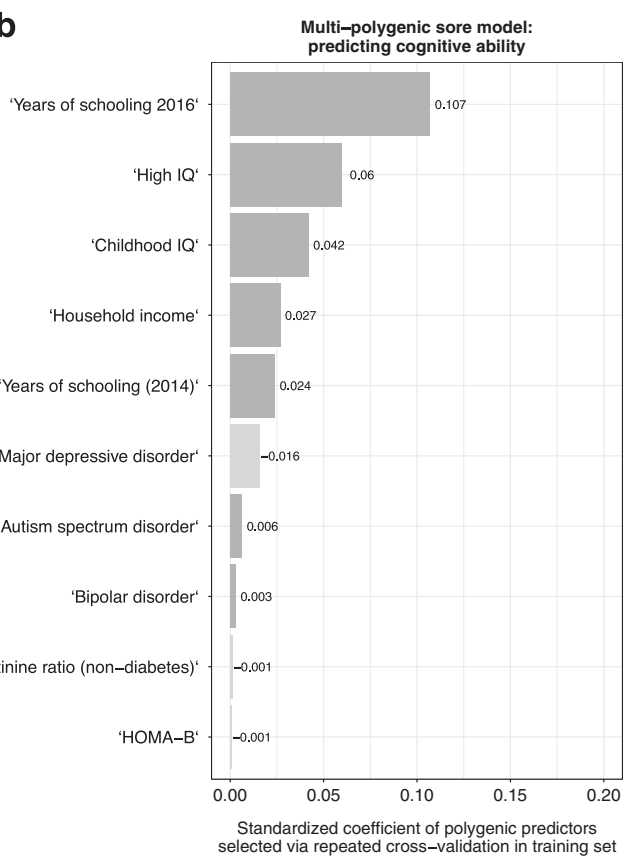

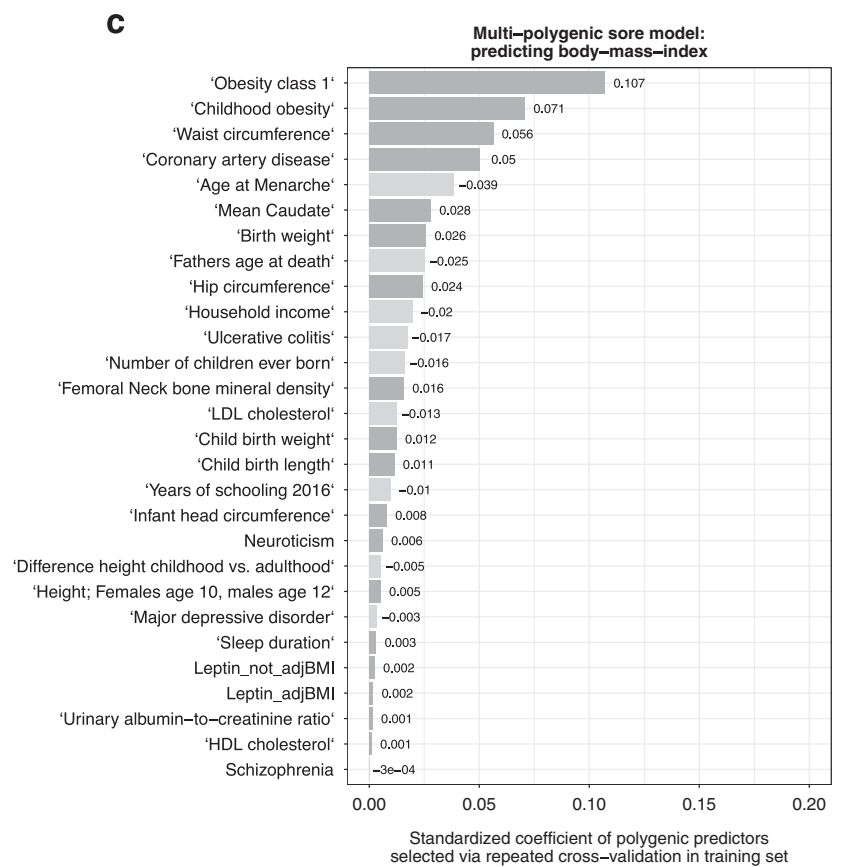

Figure 1. (a) Multi-polygenic score (MPS) model predicting educational achievement. Standardized coefficients of polygenic predictors selected by elastic net via repeated cross-validation in training set. Analogous to conventional multiple regression, a standardized coefficient represents the contribution of the predictor to the outcome when adjusting for all other variables in the model. The mean variance explained of the resampling distribution from the cross-validation was mean-cv- $R_{\text {train }}^{2}=0.12$. The out-of-sample prediction of the model was $R_{\text {test }}^{2}=0.109$. (b) MPS model predicting general cognitive ability. Standardized coefficients of polygenic predictors selected by elastic net via repeated crossvalidation in training set. Analogous to conventional multiple regression, a standardized coefficient represents the contribution of the predictor to the outcome when adjusting for all other variables in the model. The mean variance explained of the resampling distribution from the cross-validation was mean-cv- $R_{\text {train }}^{2}=0.051$. The out-of-sample prediction of the model was $R_{\text {test }}^{2}=0.048$. (c) MPS model predicting body mass index (BMI). Standardized coefficients of polygenic predictors selected by elastic net via repeated cross-validation in training set. Analogous to conventional multiple regression, a standardized coefficient represents the contribution of the predictor to the outcome when adjusting for all other variables in the model. The mean variance explained of the resampling distribution from the cross-validation was mean$\mathrm{cv}-R_{\text {train }}^{2}=0.074$. The out-of-sample prediction of the model was $R_{\text {test }}^{2}=0.054$.

random from the top $10 \%$ MPS group will have a higher score than a person picked at random from the bottom $10 \%$ group. 37,38

For cognitive ability, Figure $2 \mathrm{~b}$ illustrates that individuals in the top $10 \%$ of the MPS distribution on average had a standardized cognitive ability score over 0.64 (95\% confidence interval 0.40 0.89) s.d. higher than those in the bottom $10 \%$ MPS distribution. This means that $74 \%$ in the top $10 \%$ MPS group had mean ability score above that of the bottom $10 \%$ group, and that there is a 

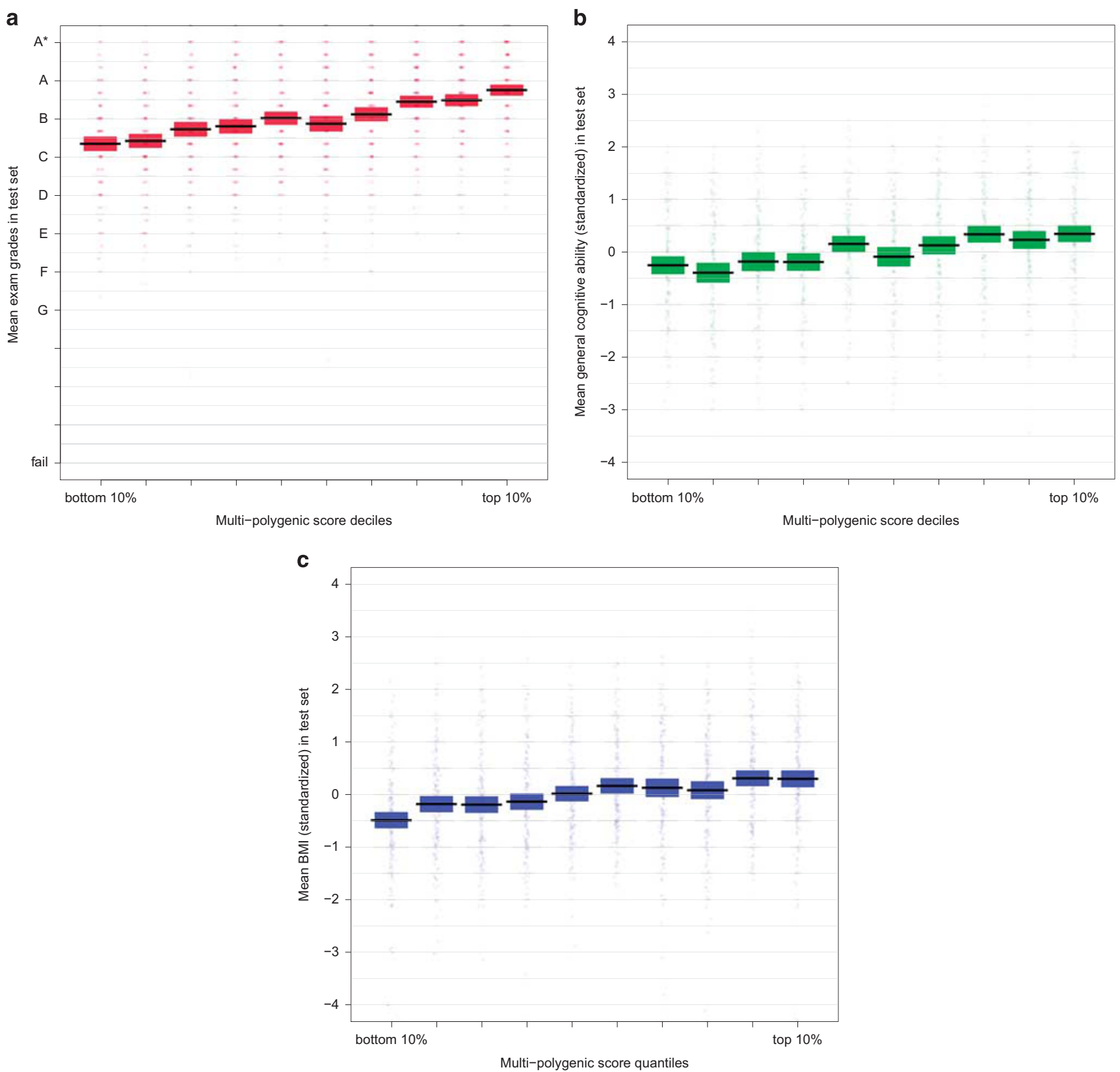

Figure 2. (a) Educational achievement by multi-polygenic score (MPS) deciles. Observed mean grade (across the three subjects Mathematics, English and Science) by deciles of the MPS predictions in the test set. Bars represent $95 \%$ confidence estimates. (b) General cognitive ability by MPS deciles. Observed mean standardized general cognitive ability by deciles of the MPS predictions in the test set. Bars represent $95 \%$ confidence estimates. (c) Body mass index (BMI) by MPS deciles. Observed mean standardized BMI (age and sex adjusted by external reference) by deciles of the MPS predictions in the test set. Bars represent $95 \%$ confidence estimates.

$67 \%$ probability that a person picked at random from the top $10 \%$ MPS group will have a higher score than a person picked at random from the bottom $10 \%$ group.

For BMI, Figure $2 \mathrm{c}$ shows that children in the top $10 \%$ of the MPS distribution on average had a 0.80 (95\% confidence interval $0.57-1.03)$ s.d. higher than those in the bottom 10\% MPS distribution. Expressed differently, $79 \%$ of children in the top $10 \%$ MPS group had a mean ability score above that of the bottom $10 \%$ group, and that there is a $71 \%$ probability that a person picked at random from the top 10\% MPS group will have a higher score than a person picked at random from the bottom $10 \%$ group.

\section{DISCUSSION}

We demonstrate that the MPS approach that combines summarylevel GWAS data from multiple traits yields better individual-level phenotype prediction than single-score predictor models in independent test data.

The observation that a multitude of polygenic scores contribute to trait prediction in the MPS models highlights the complexity of the system being studied and the somewhat arbitrary way we divide it into phenotypic characteristics. We show that polygenic variation associated with traits other than the to-be-predicted outcome contributes to prediction. For instance, although there is a known association between ulcerative colitis and $\mathrm{BMI}^{39}$ genetic 
variants associated with ulcerative colitis are not typically included in models estimating individuals' genetic risk for increased BMI.

The predictors selected and coefficients estimated by the MPS models in the current study can be used to generate individualspecific composite estimates of genetic propensities in other and smaller samples. For a more parsimonious replication, future research in other samples could construct a simple multiple regression model using the top five predictors selected by the current analyses. The predictive power of such an MPS model can then be compared with that of the best single-score model. More generally, in addition to the likely improvement in MPS prediction as more and larger GWASs are being published, the MPS approach has the potential to be applied to a wide range of outcomes and samples, including psychiatric and medical outcomes in casecontrol samples.

The predictive power of a polygenic score is not only a function of the genetic correlation between discovery and outcome trait, but also of the statistical power present in the discovery GWAS on which it is based (that is, variance explained and sample size). ${ }^{5}$ The MPS approach exploits the fact that even GWASs of genetically distantly related traits might contribute predictive power if their power is superior to GWASs of more proximal traits. For instance, most likely because of its much greater sample size, the years of education polygenic score predicted general cognitive ability better than any of the polygenic scores based on GWASs directly measuring general cognitive ability.

Because predictive power of polygenic scores does not simply reflect the genetic correlation between discovery and target trait, but depends on the genetic architecture of both traits and sample size (especially of the discovery sample), ${ }^{5,6,40}$ the MPS approach is not suited for investigating etiology. Other methods have been developed to that end. For instance, multivariate twin studies are appropriate for investigating trait etiology, or multi-trait GWAS meta-analysis aims to disentangle effects of correlated traits at the level of genetic variants. ${ }^{15,16,41-45}$ In contrast, the declared aim of the MPS approach is to maximize trait prediction, without assumptions about the relationships among predictors.

The MPS approach will be useful whenever trait prediction is a priority. The primary reason for maximizing predictive power using the MPS approach is to predict phenotypes of individuals with as much accuracy as possible. Individual-specific genetic predictions will be useful in research with modest sample sizes to investigate developmental, multivariate and gene-environment interplay issues. Eventually, MPS models could be useful in both society and science to estimate genetic potential as well as risk in relation to all domains of functioning, including cognitive abilities and disabilities, personality and health and illness.

This predictive power will raise concerns about potential early, even prenatal, prediction. It is important to begin discussions that are informed by the empirical data because genotype-based trait prediction is moving towards the point of practical relevance. Although concerns are warranted, these might be outweighed by the benefits that could result from being able to predict problems and potential early and develop stratified preventions and interventions accordingly.

\section{CONFLICT OF INTEREST}

The authors declare no conflict of interest.

\section{ACKNOWLEDGMENTS}

We gratefully acknowledge the ongoing contribution of the participants in the Twins Early Development Study (TEDS) and their families. TEDS is supported by a program grant to RP from the UK Medical Research Council (MR/M021475/1 and previously G0901245), with additional support from the US National Institutes of Health (AG046938) and the European Commission (602768; 295366). RP is supported by a Medical Research Council Professorship award (G19/2) and a European Research
Council Advanced Investigator award (295366). EK is supported by the MRC/IoPPN Excellence Award. This study presents independent research supported by the National Institute for Health Research (NIHR) Biomedical Research Centre at South London and Maudsley NHS Foundation Trust and King's College London. The views expressed are those of the author(s) and not necessarily those of the NHS, NIHR, Department of Health or King's College London. We gratefully acknowledge capital equipment funding from the Maudsley Charity (Grant Ref. 980) and Guy's and St Thomas's Charity (Grant Ref. STR130505). SN is also supported by the National Institute for Health Research (NIHR) University College London Hospitals Biomedical Research Centre, and by awards establishing the Farr Institute of Health Informatics Research at UCLPartners, from the Medical Research Council, Arthritis Research UK, British Heart Foundation, Cancer Research UK, Chief Scientist Office, Economic and Social Research Council, Engineering and Physical Sciences Research Council, National Institute for Health Research, National Institute for Social Care and Health Research and Wellcome Trust (Grant MR/K006584/1). The Supplementary Information contains extended acknowledgments.

\section{AUTHOR CONTRIBUTIONS}

Conceived and designed the experiments: EK and RP. Analyzed data and processed and quality controlled genotype data: EK. Performed/supervised manual quality control and calling of genotype data: HP, SN and CJC. Wrote the paper: EK and RP. All authors contributed to and critically reviewed the manuscript.

\section{REFERENCES}

1 Eicher JD, Landowski C, Stackhouse B, Sloan A, Chen W, Jensen N et al. GRASP v2.0: an update on the Genome-Wide Repository of Associations between SNPs and phenotypes. Nucleic Acids Res 2015; 43: D799-D804.

2 Visscher PM, Brown MA, McCarthy MI, Yang J. Five years of GWAS discovery. Am J Hum Genet 2012; 90: 7-24.

3 Wood AR, Esko T, Yang J, Vedantam S, Pers TH, Gustafsson S et al. Defining the role of common variation in the genomic and biological architecture of adult human height. Nat Genet 2014; 46: 1173-1186.

4 Locke AE, Kahali B, Berndt SI, Justice AE, Pers TH, Day FR et al. Genetic studies of body mass index yield new insights for obesity biology. Nature 2015; 518: 197-206.

5 Dudbridge F. Power and predictive accuracy of polygenic risk scores. PLoS Genet 2013; 9: e1003348.

6 Palla L, Dudbridge F. A fast method that uses polygenic scores to estimate the variance explained by genome-wide marker panels and the proportion of variants affecting a trait. Am J Hum Genet 2015; 97: 250-259.

7 Euesden J, Lewis CM, O'Reilly PF. PRSice: polygenic risk score software. Bioinformatics 2014; 31: 1466-1468, btu848.

8 Visscher PM, Yang J. A plethora of pleiotropy across complex traits. Nat Genet 2016; 48: 707-708.

9 Bulik-Sullivan B, Finucane HK, Anttila V, Gusev A, Day FR, Loh P-R et al. An atlas of genetic correlations across human diseases and traits. Nat Genet 2015; 47: 1236-1241.

10 Pickrell JK, Berisa T, Liu JZ, Ségurel L, Tung JY, Hinds DA. Detection and interpretation of shared genetic influences on 42 human traits. Nat Genet 2016; 48: 709-717.

11 Krapohl E, Euesden J, Zabaneh D, Pingault J-B, Rimfeld K, von Stumm S et al. Phenome-wide analysis of genome-wide polygenic scores. Mol Psychiatry 2016; 21: 1188-1193.

12 Solovieff N, Cotsapas C, Lee PH, Purcell SM, Smoller JW. Pleiotropy in complex traits: challenges and strategies. Nat Rev Genet 2013; 14: 483-495.

13 Zou H, Hastie T. Regularization and variable selection via the elastic net. J R Stat Soc Ser B Stat Methodol 2005; 67: 301-320.

14 Maier R, Moser G, Chen G-B, Ripke S, Cross-Disorder Working Group of the Psychiatric Genomics Consortium, Coryell W et al. Joint analysis of psychiatric disorders increases accuracy of risk prediction for schizophrenia, bipolar disorder, and major depressive disorder. Am J Hum Genet 2015; 96: 283-294.

15 Baselmans BM, Jansen R, Dongen J, van, Bao Y, Smart M, Kumari M et al. Multivariate genome-wide and integrated transcriptome and epigenome-wide analyses of the well-being spectrum. bioRxiv 2017; doi: 10.1101/115915.

16 Ferreira MAR, Purcell SM. A multivariate test of association. Bioinformatics 2009; 25: 132-133.

17 Turley P, Walters RK, Maghzian O, Okbay A, Lee JJ, Fontana MA et al. MTAG: multi-trait analysis of GWAS. bioRxiv 2017; doi: 10.1101/118810.

18 Selzam S, Krapohl E, von Stumm S, O'Reilly PF, Rimfeld K, Kovas Y et al. Predicting educational achievement from DNA. Mol Psychiatry 2017; 22: 267-272. 
19 Krapohl E, Plomin R. Genetic link between family socioeconomic status and children's educational achievement estimated from genome-wide SNPs. Mol Psychiatry 2016; 21: 437-443.

20 Kovas Y, Haworth CMA, Dale PS, Plomin R. The genetic and environmental origins of learning abilities and disabilities in the early school years. Monogr Soc Res Child Dev 2007; 72, vii 1-144.

21 McCarthy S, Das S, Kretzschmar W, Durbin R, Abecasis G, Marchini J. A reference panel of 64,976 haplotypes for genotype imputation. bioRxiv 2015; 48: 1279-1283, 35170.

22 Zheng J, Erzurumluoglu AM, Elsworth BL, Kemp JP, Howe L, Haycock PC et al. LD Hub: a centralized database and web interface to perform LD score regression that maximizes the potential of summary level GWAS data for SNP heritability and genetic correlation analysis. Bioinformatics 2017; 33: 272-279.

23 Bulik-Sullivan BK, Loh P-R, Finucane HK, Ripke S, Yang J, Schizophrenia Working Group of the Psychiatric Genomics Consortium et al. LD score regression distinguishes confounding from polygenicity in genome-wide association studies. Nat Genet 2015; 47: 291-295.

24 Vilhjálmsson BJ, Yang J, Finucane HK, Gusev A, Lindström S, Ripke S et al. Modeling linkage disequilibrium increases accuracy of polygenic risk scores. Am J Hum Genet 2015; 97: 576-592.

25 Kuhn M. Building predictive models in R using the caret package. J Stat Softw 2008; 28: 1-26.

26 Tibshirani R. Regression shrinkage and selection via the Lasso. J R Stat Soc Ser B Methodol 1996; 58: 267-288.

27 Zhou D-X. On grouping effect of elastic net. Stat Probab Lett 2013; 83: 2108-2112.

28 Friedman J, Hastie T, Tibshirani R. Regularization paths for generalized linear models via coordinate descent. J Stat Softw 2010; 33: 1-22.

29 Kohavi R. A study of cross-validation and bootstrap for accuracy estimation and model selection. In: Proceedings of the 14th International Joint Conference on Artificial Intelligence - Volume 2. Morgan Kaufmann Publishers Inc.: San Francisco, CA, USA, 1995 (cited 14 December 2016), pp 1137-1143. Available from http://dl. acm.org/citation.cfm? $\mathrm{id}=1643031.1643047$.

30 Efron B. Estimating the error rate of a prediction rule: improvement on cross-validation. J Am Stat Assoc 1983; 78: 316-331.

31 Efron B, Tibshirani R. Improvements on cross-validation: the $632+$ bootstrap method. J Am Stat Assoc 1997; 92: 548-560.

$32 \mathrm{Kim} \mathrm{J-H}$. Estimating classification error rate: repeated cross-validation, repeated hold-out and bootstrap. Comput Stat Data Anal 2009; 53: 3735-3745.

33 Malenka RC, Nestler E, Hyman S, Sydor A, Brown R. Molecular Neuropharmacology: A Foundation for Clinical Neuroscience. McGraw Hill Medical Book: New York, 2009.

34 Aron AR, Schlaghecken F, Fletcher PC, Bullmore ET, Eimer M, Barker R et al. Inhibition of subliminally primed responses is mediated by the caudate and thalamus: evidence from functional MRI and Huntington's disease. Brain 2003; 126: $713-723$.
35 Jahanshahi M, Obeso I, Rothwell JC, Obeso JA. A fronto-striato-subthalamicpallidal network for goal-directed and habitual inhibition. Nat Rev Neurosci 2015; 16: 719-732.

36 Seger CA, Cincotta CM. The roles of the caudate nucleus in human classification learning. J Neurosci 2005; 25: 2941-2951.

37 Ruscio J. A probability-based measure of effect size: robustness to base rates and other factors. Psychol Methods 2008; 13: 19-30.

38 Cohen J. Statistical Power Analysis for the Behavioral Sciences, 2nd edn. Academic Press: New York, NY, US, 1988.

39 Dong J, Chen Y, Tang Y, Xu F, Yu C, Li Y et al. Body mass index is associated with inflammatory bowel disease: a systematic review and meta-analysis. PLOS ONE 2015; 10: e0144872.

40 Wray NR, Lee SH, Mehta D, Vinkhuyzen AAE, Dudbridge F, Middeldorp CM. Research review: polygenic methods and their application to psychiatric traits. J Child Psychol Psychiatry 2014; 55: 1068-1087.

41 Majumdar A, Haldar T, Bhattacharya S, Witte J. An efficient Bayesian meta-analysis approach for studying cross-phenotype genetic associations. bioRxiv. 2017; doi: $10.1101 / 101543$

42 Zhu X, Feng T, Tayo BO, Liang J, Young JH, Franceschini N et al. Meta-analysis of correlated traits via summary statistics from GWASs with an application in hypertension. Am J Hum Genet 2015; 96: 21-36.

43 Bhattacharjee S, Rajaraman P, Jacobs KB, Wheeler WA, Melin BS, Hartge P et al. A subset-based approach improves power and interpretation for the combined analysis of genetic association studies of heterogeneous traits. Am J Hum Genet 2012; 90: 821-835.

44 Shim H, Chasman DI, Smith JD, Mora S, Ridker PM, Nickerson DA et al. A multivariate genome-wide association analysis of 10 LDL subfractions, and their response to statin treatment, in 1868 Caucasians. PLOS ONE 2015; 10: e0120758.

45 Bolormaa S, Pryce JE, Reverter A, Zhang Y, Barendse W, Kemper $\mathrm{K}$ et al. A multi-trait, meta-analysis for detecting pleiotropic polymorphisms for stature, fatness and reproduction in beef cattle. PLOS Genet 2014; 10: e1004198.

(i) This work is licensed under a Creative Commons Attribution 4.0 International License. The images or other third party material in this article are included in the article's Creative Commons license, unless indicated otherwise in the credit line; if the material is not included under the Creative Commons license, users will need to obtain permission from the license holder to reproduce the material. To view a copy of this license, visit http://creativecommons.org/licenses/ by/4.0/

(c) The Author(s) 2018

Supplementary Information accompanies the paper on the Molecular Psychiatry website (http://www.nature.com/mp) 